strength means that electrons cannot be regarded ontircly as non-relativistic particles, as required in the cyclotron theory.

If the radiation is cyclotron radiation from elcctrons, the frequency of generation is $\beta^{-2}$ times the observed frequency. 'Thus the radiation at $408 \mathrm{MHz}$ would be generated at $13 \mathrm{MHz}$, corresponding to a field strength of 5 Oersted. The energy density of the static magnetic field therefore seems to be less than the energy in the total radiation field, which again leads one to suspect that the simple cyclotron theory does not provide a sufficient explanation.

We may conclude that: (i) the beaming of pulsar radiation is due to the relativistic effect of rapid rotation of the source, (ii) the radiation mechanism is roughly isotropic, (iii) the polarization follows the pattern of cyclotron radiation, (iv) if electron cyclotron radiation is to provide an explanation, a non-linear theory must be developed to account for the high radiated field strengths.

I thank D. A. Graham for help with the observations, and Professor F. D. Kahn for a discussion of the radiation mechanism.

University of Manchester,

F. G. SмIтн

Nuffield Radio Astronomy Laboratories,

Jodrell Bank.

Received July 11, 1969

${ }^{1}$ (lark, R. R., and Smith, F. G., Nature, 221, 724 (1969).

${ }^{2}$ Gold, T., Nature, 218, 731 (1968).

\section{Magnetic Fields and Highly Condensed Objects}

Iт has become conventionel in astrophysies to aceept the widespread presence of magnetic fields, but not to worry much about their origin. Because of the very long time scales for ohmic decay in most objects it is usual to ascribe the present magnetic field to a field thet existed at cerlier epochs. By repetition of this process one can trace the fields back to the beginning of a big-bang universe-for example, one attributes the magnetic fiolds of stars to that which existed in the ges cloud from which the stars condensed, the fields in such clouds to the galaxy containing them, the field of the galaxy to the universe, and the latter to the first moment of time. Then one need no longer trouble oncself with the problem, which is convenient because the problem is otherwise awkward: a voltage $\sim 10^{14} \mathrm{~V}$ would have to be applied across the galaxy for $10^{10} \mathrm{yr}$ in order to build a galactic field of $\sim 10^{-5} \mathrm{G}$.

Recently, it has become clear that highly condensed objects are important energy sources, and the question evidently arises as to whether they augment the galactic field by dynamo action. This could happen through the winding by rotation of a field that emerges from the object. Consider an object of mass $M$ with a surface field $H_{\mathrm{s}}$. Let the rotary energy converted to magnetic energy be written as $f M c^{2}$. The fraction $f$ of the rest mass involved in this way may be quite large, $>10^{-1}$ say for a sufficiently condensed object. The equation

$$
f M c^{2}=\left(H_{\mathrm{s}}{ }^{2} / 8 \pi\right) V
$$

can be interpreted as meaning that the rotary energy converted to magnetic energy is sufficient to fill a box of volume $V$ with a field of intensity $H_{\mathrm{s}}$.

The volume $V$ given by equation (1) is small, for any reasonable values of $M, H_{s}$, compared with the volume $V_{\mathrm{g}} \simeq 10^{67} \mathrm{~cm}^{3}$ of the disk of the galaxy. Hence to obtain a galactic magnetic field we must next imagine the box
$V$ to be expanded adiabatically to volume $V_{\mathrm{g}}$. The resulting field intensity $H$ is given by

$$
H \simeq H_{\mathrm{s}}\left(V / V_{\mathrm{g}}\right)^{2 / 3}
$$

Eliminating $V$ between equations (1) and (2) leads to

$$
H \simeq H_{\mathrm{s}}{ }^{-1 / 3}\left(8 \pi f M c^{2} / V_{\mathrm{g}}\right)^{2 / 3}
$$

With $M=10^{9} \quad M \circ, f=10^{-1}, \quad V_{\mathrm{g}}=10^{67} \mathrm{~cm}^{3}, H_{\mathrm{s}}=10^{9} \mathrm{G}$, equation (3) gives $H \simeq 10^{-5} \mathrm{G}$. In our own galaxy $M \simeq 10^{8} M_{\odot}$ would perhaps be a better estimate for the mass of the extreme nucleus. We might therefore expect that the overall galactic magnetic field that could be produced by such a nucleus would be somewhat less than $10^{-5} \mathrm{G}$. On the other hand, $H_{\mathrm{s}} \simeq 10^{9} \mathrm{G}$ is close to the maximum permitted by the gravitational stability of the object, and decreasing $H_{\mathrm{s}}$ increases $H$, although not sensitively. Evidently $H \simeq 10^{-5} \mathrm{G}$ is not much affected by uncertainties in $M, H_{\mathrm{s}}$, and this estimate is indeed close to the actual field of the galaxy.

It is of interest to consider a very different case, appropriate to the Crab nebula. With $M=M_{\odot}, f=10^{-1}, V_{\mathrm{g}}$ taken now as $\sim 10^{55} \mathrm{~cm}^{3}, H_{\mathrm{s}} \simeq 10^{9} \mathrm{G}$, equation (3) gives $H \simeq 10^{-3}(\mathrm{x}$. This is again close to estimates for the cctual field of the Crab. It therefore seems possible that the magnetic field of the Crab nebula might have been produced by the ecntral object-if the original rotary energy was large enough.

While the present process does not explain the origin of an initial field, it permits amplification of an initial field. We have to consider the ficld in two stages. Starting from diffuse material of density $\rho_{i}$ in the galaxy, and from an initial field intensity $H_{\mathrm{i}}$, we form a condensed object of density $\rho$ and field $H_{s}$. For isotropic condensation

$$
H_{\mathrm{s}} \simeq H_{\mathrm{i}}\left(\rho / \rho_{\mathrm{i}}\right)^{2 / 3}
$$

For a highly compact object near its Schwarzschild radius $\rho$ is related to $M$ by

$$
p \simeq 10^{16}\left(M_{\odot} / M\right)^{2} \mathrm{~g} \mathrm{~cm}^{-3}
$$

Taking $\rho_{\mathrm{i}} \simeq 10^{-24} \mathrm{~g} \mathrm{~cm}^{-3}$ in the gelexy, and using equation (5), we have from equation (4)

$$
H_{\mathrm{s}} \simeq\left(10^{20} M_{\odot} / M\right)^{4 / 3} H_{\mathrm{i}}
$$

Putting $f \simeq 10^{-1}, \quad V_{\mathrm{g}} \simeq 10^{67} \mathrm{~cm}^{3}$ in equation (3), and using equation. (6) for $\bar{H}_{\mathrm{s}}$, it is easy to see that

$$
\frac{H}{H_{\mathrm{i}}} \simeq 10^{-17} H_{\mathrm{i}}^{-4 / 3}\left(\frac{M}{M_{O}}\right)^{10 / 9}
$$

and hence that the field is amplificd provided the right hand side of equation (7) is greater than unity, that is, provided

$$
H_{\mathrm{i}}<\simeq 10^{-1 \mathrm{a}}\left(M / M_{\odot}\right)^{5 / 6} \mathrm{G}
$$

For $M / M_{\odot} \simeq 10^{9}$, expression (8) gives $H_{\mathrm{i}}<\sim 3 \times 10^{-6} \mathrm{G}$, which is close to the actual field of the galaxy. The fact that the actual field agrees with the limit set by expression (8) suggests that field amplification in the galaxy is now essentially complete.

Finally, we note that many small masses are less efficient than one object of the same total mass unless the fields produced by the small masses have the same sense.

Institute of Theoretical Astronomy,

F. HOYLE

University of Cambridge.

Received July 23, 1969.

\section{Solar Wind Tail and the Anisotropic Production of Fast Hydrogen Atoms}

THe flow of supersonic solar wind is supposed to be reduced to subsonic velocities and randomized at a so.called shock front somewhere between 5 and $50 \mathrm{AU}$ from the Sun. 